\title{
CONVOLUTION THEOREMS RELATED WITH THE SOLVABILITY OF WIENER-HOPF PLUS HANKEL INTEGRAL EQUATIONS AND SHANNON'S SAMPLING FORMULA
}

\author{
LUÍS PINHEIRO CASTRO*, RITA CORREIA GUERRA* AND NGUYEN MINH TUAN**
}

\begin{abstract}
This paper considers two finite integral transforms of Fourier-type, in view to propose a set of eight new convolutions, and to analyze the solvability of a class of the integral equations of Wiener-Hopf plus Hankel type, defined on finite intervals, which is involved in engineering problems. The solvability and solution of the considered equations are investigated by means of Fourier-type series, and a Shannon-type sampling formula is obtained. Some concluding remarks with respect to theoretical issues and engineering applications are emphasized in the last section, along with the analysis of some illustrative cases, which exemplify that the present method solves cases which are not under the conditions of previously known techniques.
\end{abstract}

\section{IntRoduction}

Convolutions and integral equations of convolution-type occupy a central position both in mathematical theoretical studies and in applications to other sciences. Indeed, on the one hand convolutions are effectively applied in many practical problems (even outside mathematics), on the other hand each convolution is itself a new integral transform and so subjected to a mathematical theoretical analysis (cf., e.g., 2, 4, 6, 7, 10, 11, 8, 9]). For instance, the Hilbert and Cauchy integral transforms can be interpreted as convolutions in some sense. This is why despite decades of research, convolutions and their associated equations continue to be vigorously developed by many authors, and time-to-time become powerful tools in engineering applications (see [3, 13, 18] and references therein). For the convenience of presenting our motivation in more detail, we start by recalling the Fredholm integral equation

$$
\lambda \varphi(x)+\int_{0}^{2 \pi} K(x, u) \varphi(u) d u=f(x),
$$

where $\varphi$ is unknown, $\lambda \in \mathbb{C}, K(x, y):=p(x-u)+q(x+u)$, with $p, q$ and $f$ being given elements. The above equation is called of Wiener-Hopf plus Hankel type due to the structure of its kernel, as we may recognize in the second left-hand side term of the equation. There exists a long list of works concerning particular cases in which the kernel has just the Wiener-Hopf component or only the Hankel one (i.e., $q$ ).

2010 Mathematics Subject Classification. Primary 42A85; Secondary 35S30, 45E10, 47B35.

Key words and phrases. Convolution, integral equation, factorization, Fourier integral operator, Wiener-Hopf operator, Hankel operator.

†Accepted author's manuscript (AAM) of [L. P. Castro, R. C. Guerra, N. M. Tuan: Mathematica Slovaca, Volume 69, Number 5 (2019), 1149-1164. DOI: 10.1515/ms-2017-0297]. The final publication is available at https: //doi.org/10.1515/ms-2017-0297 This work was supported in part by FCT-Portuguese Foundation for Science and Technology through the Center for Research and Development in Mathematics and Applications (CIDMA) of Universidade de Aveiro, within project UID/MAT/04106/2019, and by the Viet Nam National Foundation for Science and Technology Development (NAFOSTED). R. C. Guerra also acknowledges the direct support of the Portuguese Foundation for Science and Technology (FCT) through the scholarship PD/BD/114187/2016. 
One of the main reasons for the existence of such studies is that the solutions of those equations have many useful applications in diverse fields such as: scattering theory, fluid dynamics, filtering theory, circular punch penetration in finitely thick elastic layers, and rarefied gas dynamics. Equation (1.1) also arises in linear filtering of stationary random processes, and in the field of fast signal processing. Such equation arises often from one of two possible ways: the first is from the modeling situations in terms of differential operators/equations, and the second one is from the so-called convolution integral problems (see [1, 5, 15, 19] and references therein). There are also studies of (1.1) when $K(x, y)$ is generated by a single function, i.e., $K(x, u)=p(x-u)+p(x+u)$. However, the complexity is much greater when studying the above equation for kernels $K(x, y)$ generated by two distinct functions $p$ and $q$ which are defined on a finite interval. In particular, the approach to equation (1.1) and its solvability can be totally different, being dependent mainly on the attributes of the given data $p, q, f$, and on the type of used extensions for $p, q, f$ outside the domain (odd, even, periodic extensions, etc.).

Motivated by the periodicity and oscillation nature of certain integrals, our approach to analyze equation 1.1 comes from Fourier series and integral transforms on finite intervals, which is different e.g. from the approach via the resolvent kernel of $K(x, y)$ given by Tsitsiklis in [19]. Namely, this work is devoted to two finite integral transforms of Fourier-type, series of Fourier-type, a set of eight new convolutions appropriately constructed, and the $L^{2}$-solution of the equation 1.1 which can be seen as a resulting goal of the previously constructed issues. Regarding the convolutions, in some other studies, there is the application of other methods with weighted Lebesgue spaces in which is possible to obtain norm inequalities among some $L^{p}$-spaces. This is the case of the works [17, 12, 14, in which, at least in one of them, are also used methods of reproducing kernel Hilbert spaces. The present work proposes a different approach although containing some of the goals of those other works. Note that knowledge about the solvability of the above integral equation may have some applications in particular problems of linear time-invariant systems. This paper is divided into six sections and organized as follows.

Section 2 presents a general framework on the finite integral transforms here considered and on series of Fourier-type. In Section 3, we provide a set of eight convolutions associated with those transform and series. These are absolutely new convolutions which cannot be derived from any previously known convolutions. In Section 4 a special attention is devoted to the use of the constructed convolutions and transforms for the solvability of integral equations of Wiener-Hopf plus Hankel type. In Section 5 we exemplify the solvability and exhibit the solution of some particular equations which cannot be worked out by using previously published works. Section 6 is just a sort of a theoretical concluding remark by stating a proposition which involves potential practical applications and a theorem about theoretical consequences on a class of Fredholm operators in the $L^{2}$-Hilbert space framework.

\section{Finite integral transforms And SERIEs of Fourier-type}

The Fourier series and its variations play a central role in harmonic analysis. There exist many different ways of defining Fourier series, all of which are consistent with one another, but each of which emphasizes some particular aspects of the series (see [20]). This section presents two finite integral transforms of Fourier-type and their associated series. We begin with the basic definition of the Fourier series.

Definition 1 (see [13, 18]). Let $f$ be a Lebesgue integrable function on a finite interval $[0,2 \pi]$. The infinite sum

$$
\left(F_{f}\right)(x):=\frac{\hat{f}_{c}(0)}{2}+\sum_{n=1}^{\infty}\left[\hat{f}_{c}(n) \cos (n x)+\hat{f}_{s}(n) \sin (n x)\right]
$$


is called the Fourier series of $f$ on $[0,2 \pi]$, where $\hat{f}_{c}(n), \hat{f}_{s}(n)$ are the Fourier coefficients of $f$ defined by

$$
\begin{aligned}
& \hat{f}_{c}(n)=\frac{1}{\pi} \int_{0}^{2 \pi} \cos (n x) f(x) d x, n \in \mathbb{N}, \\
& \hat{f}_{s}(n)=\frac{1}{\pi} \int_{0}^{2 \pi} \sin (n x) f(x) d x, n \in \mathbb{N} .
\end{aligned}
$$

At this point, we say nothing about the convergence of the series (2.1). Namely, it is well-known that there are functions $f \in L^{1}([0,2 \pi])$ such that their associated series $(2.1)$ diverge at every point $x \in[0,2 \pi]$. However, if $1<p<+\infty$ and if $f \in L^{p}([0,2 \pi])$, then its series (2.1) converges in mean ( $L^{p}$-norm) to a function in $L^{p}([0,2 \pi])$. In the framework of this paper, the convergence is in mean of the Lebesgue space $L^{p}([0,2 \pi])$, with $p>1$ (see [13, 16, 18]). Let us write

$$
\mathscr{E}(x):=\frac{2 \cos (x)+i \sin (x)}{\sqrt{5 \pi}} .
$$

The series 2.1 may be rewritten as follows

$$
\begin{aligned}
\left(F_{f}\right)(x) & =\frac{\hat{f}_{c}(0)}{2}+\sum_{n=1}^{\infty}\left\{\frac{\sqrt{5 \pi}}{2}\left(\frac{1}{2} \hat{f}_{c}-i \hat{f}_{s}\right)(n) \mathscr{E}(n x)\right. \\
& \left.+\frac{\sqrt{5 \pi}}{2}\left(\frac{1}{2} \hat{f}_{c}+i \hat{f}_{s}\right)(n) \mathscr{E}(-n x)\right\}=\frac{\hat{f}_{c}(0)}{2}+\sum_{0 \neq n=-\infty}^{+\infty} a_{n} \mathscr{E}(n x),
\end{aligned}
$$

where

$$
a_{n}=\left\{\begin{array}{lll}
\frac{\sqrt{5 \pi}}{2}\left(\frac{1}{2} \hat{f}_{c}(n)+i \hat{f}_{s}\right)(n) & \text { if } & n<0 \\
\frac{\sqrt{5 \pi}}{4} \hat{f}_{c}(n) & \text { if } & n=0 \\
\frac{\sqrt{5 \pi}}{2}\left(\frac{1}{2} \hat{f}_{c}(n)-i \hat{f}_{s}\right)(n) & \text { if } & n>0
\end{array}\right.
$$

We will be using the usual inner product in $L^{2}([0,2 \pi])$. Namely, for any $f$ and $g$ in $L^{2}([0,2 \pi])$ their inner product is given by

$$
\langle f, g\rangle_{2}=\int_{0}^{2 \pi} f(x) \bar{g}(x) d x .
$$

The expression (2.4 leads us to the following proposition which is a direct consequence of the complete orthonormal basis $\left\{e^{i n x}\right\}_{n \in \mathbb{Z}}$ of the Hilbert space $L^{2}([0,2 \pi])$.

Proposition 2.1. The set of functions

$$
\left\{\frac{1}{\sqrt{2 \pi}}, \mathscr{E}(n x), n \in \mathbb{Z} \backslash\{0\}\right\}
$$

is a complete orthonormal basis of $L^{2}([0,2 \pi])$.

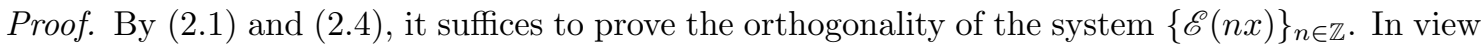
of this, having in mind the usual inner product in $L^{2}([0,2 \pi])$, we compute

$$
\begin{aligned}
\langle\mathscr{E}(n x), \mathscr{E}(m x)\rangle_{2} & =\frac{1}{5 \pi} \int_{0}^{2 \pi}(2 \cos (n x)+i \sin (n x))(2 \cos (m x)-i \sin (m x)) d x \\
& = \begin{cases}0, & n \neq m \\
1, & n=m,\end{cases}
\end{aligned}
$$

for $n, m \neq 0$. 
For $n=0$, we have

$$
\left\langle\frac{1}{\sqrt{2 \pi}}, \frac{1}{\sqrt{2 \pi}}\right\rangle_{2}=\frac{1}{2 \pi} \int_{0}^{2 \pi} 1 d x=1
$$

The proposition is proved.

In what follows, the notation $\left(F_{f}\right)(x)$ concerning the Fourier series will be replaced by $\mathcal{E}_{f}(x)$ given by (2.4).

Many other Fourier-related integral transforms have been defined, extending the initial idea to more suitable forms for concrete applications (e.g. in engineering problems). In particular, the associated Fourier series have a great number of applications in practical problems [13, 18. The representation in the form (2.4) naturally suggests us to define a finite Fourier-type transform as follows.

Definition 2 (Finite Fourier-type transforms). (a) Let $f$ be a Lebesgue integrable function on $[0,2 \pi]$. The finite Fourier-type transform of $f$ corresponding to $\mathscr{E}(n x)$ is defined by

$$
\left(T_{1} f\right)(n):=\frac{1}{2} \sqrt{\frac{5}{\pi}} \int_{0}^{2 \pi}\left(\frac{1}{2} \cos (n x)-i \sin (n x)\right) f(x) d x, n \in \mathbb{Z} .
$$

(b) The infinite sum

$$
\left(\mathcal{E}_{f}\right)(x):=\sum_{n \in \mathbb{Z}}\left(T_{1} f\right)(n) \mathscr{E}(n x)
$$

is called finite Fourier-type series of $f$ on $[0,2 \pi]$ corresponding to $\mathscr{E}(n x)$.

Let us write

$$
\ell_{2}:=\left\{\left\{s_{n}\right\}_{n}: s_{n} \in \mathbb{C} \text { with } \sum_{n \in \mathbb{Z}}\left|s_{n}\right|^{2}<\infty\right\},
$$

which stands for the Hilbert space of all convergent-square complex series.

Let us recall the known Riesz-Fischer theorem, which states that a measurable function on $[-\pi, \pi]$ is square integrable if and only if the corresponding Fourier series converges (in the space $\left.L^{2}\right)$. In other words, for any $f \in L^{2}(-\pi, \pi)$ and its $n$-th Fourier coefficient given by

$$
F_{n}=\frac{1}{2 \pi} \int_{-\pi}^{\pi} f(x) e^{-i n x} d x \quad(n \in \mathbb{Z}),
$$

we have

$$
f(x)=\sum_{n \in \mathbb{Z}} F_{n} e^{i n x} \quad \text { for almost every } x \in(-\pi, \pi) ;
$$

conversely, for any $\left\{a_{n}\right\}_{n=-\infty}^{n=+\infty} \in \ell_{2}$, there exists a function $f \in L^{2}(-\pi, \pi)$ such that the values $a_{n}$ are the Fourier coefficients of $f$. In view of this, the integral transform 2.10 defines an invertible bounded linear operator between two Hilbert spaces $L^{2}(\pi, \pi)$ and $\ell_{2}$ in which the inversion formula is given by (2.11). Loosely speaking, the kernel $e^{-i n x}$ appears in 2.10 according to the Fourier basis $e^{i n x}$ in the inversion formula (2.11).

Remark 1. The above consideration of $T_{1}$ is natural for the extension $(2.9)$, as $\frac{1}{2} \cos (n x)-i \sin (n x)$ is the inversion kernel of the basis $2 \cos (n x)+i \sin (n x)=\sqrt{5 \pi} \mathscr{E}(n x)$ (see [6]).

Put

$$
\begin{aligned}
T_{1}: L^{2}([0,2 \pi]) & \longrightarrow \ell_{2} \\
f & \longmapsto\left\{T_{1} f\right\}_{n \in \mathbb{Z}} .
\end{aligned}
$$

By 2.4, 2.8, and 2.9 we can state the following theorem. 
Theorem 2.2. The integral transform (2.8) defines a bounded linear map from $L^{2}(-\pi, \pi)$ to $\ell_{2}$ whose inversion map is given by 2.9.

The following propositions are immediate consequences of the finite Fourier integral transform case.

Proposition 2.3 (Uniqueness theorem). If $f \in L^{1}([0,2 \pi])$, with $\left(T_{1} f\right)(n)=0$, for all $n \in \mathbb{Z}$, then $f \equiv 0$ (in $L^{1}$-norm).

Proposition 2.4 (Riemann-Lebesgue lemma). If $f \in L^{1}([0,2 \pi])$, then

$$
\lim _{n \rightarrow \infty}\left(T_{1} f\right)(n)=0 .
$$

For a certain purpose (as we will see in the next section), we also define

$$
\left(T_{2} f\right)(n):=\frac{1}{2} \sqrt{\frac{5}{\pi}} \int_{0}^{2 \pi}\left(\frac{1}{2} \cos (n x)+i \sin (n x)\right) f(x) d x, n \in \mathbb{Z} .
$$

Since $T_{2}(n)=T_{1}(-n)$, for every $n \in \mathbb{Z}$, the above propositions also obviously work for the transform $T_{2}$.

\section{Convolutions associated With $T_{1}$ And $T_{2}$}

In the present section we construct a set of eight new generalized convolutions associated with $T_{1}$ and $T_{2}$, and prove some norm inequalities which we realize to be fundamental in view of their analysis within Lebesgue spaces.

Definition 3. For any two functions $f, g$ that are Lebesgue integrable on $[0,2 \pi]$, being $f$ a function defined on $\mathbb{R}$ and $2 \pi$-periodic, we define four finite Fourier-type convolutions as follows:

$$
\begin{aligned}
&\left(f \underset{T_{1}}{*} g\right)(x):=\frac{1}{16} \sqrt{\frac{5}{\pi}} \int_{0}^{2 \pi}[7 f(x-v)- f(x+v)-3 f(-x+v) \\
&+3 f(-x-v)] g(v) d v ; \\
&\left(f \underset{T_{1}, T_{1}, T_{2}}{*} g\right)(x):=\frac{1}{16} \sqrt{\frac{5}{\pi}} \int_{0}^{2 \pi}[-3 f(x-v)+ 7 f(x+v)+3 f(-x+v) \\
&-3 f(-x-v)] g(v) d v ; \\
&\left(f \underset{T_{1}, T_{2}, T_{1}}{*} g\right)(x):=\frac{1}{16} \sqrt{\frac{5}{\pi}} \int_{0}^{2 \pi}[-3 f(x-v)+3 f(x+v)+7 f(-x+v)-3 f(-x-v)] g(v) d v ; \\
&\left(f \underset{T_{1}, T_{2}, T_{2}}{*} g\right)(x):=\frac{1}{16} \sqrt{\frac{5}{\pi}} \int_{0}^{2 \pi}[3 f(x-v)-3 f(x+v)-3 f(-x+v) \\
&+7 f(-x-v)] g(v) d v .
\end{aligned}
$$

The next theorem clarifies the relation of those convolutions with the previously considered operators.

Theorem 3.1 (Convolution theorem). If $f, g$ are Lebesgue integrable on $[0,2 \pi]$ and $f$ is a function defined on $\mathbb{R}$ and $2 \pi$-periodic, then each one of the multiplications introduced in Definition 3 has its factorization identity associated with the integral operators $T_{1}, T_{2}$ and the corresponding norm inequality:

$$
\left(T_{1}\left(f \underset{T_{1}}{*} g\right)\right)(n)=\left(T_{1} f\right)(n)\left(T_{1} g\right)(n) \text { for } n \in \mathbb{Z},
$$




$$
\begin{aligned}
\left\|f \underset{T_{1}}{*} g\right\|_{1} & \leq \sqrt{\frac{5}{\pi}}\|f\|_{1}\|g\|_{1} ; \\
\left(T_{1}\left(f \underset{T_{1}, T_{1}, T_{2}}{*} g\right)\right)(n) & =\left(T_{1} f\right)(x)\left(T_{2} g\right)(n) \text { for } n \in \mathbb{Z}, \\
\left\|f \underset{T_{1}, T_{1}, T_{2}}{*} g\right\|_{1} & \leq \sqrt{\frac{5}{\pi}}\|f\|_{1}\|g\|_{1} ; \\
\left(T_{1}\left(f_{T_{1}, T_{2}, T_{1}}^{*} g\right)\right)(n) & =\left(T_{2} f\right)(x)\left(T_{1} g\right)(n) \text { for } n \in \mathbb{Z}, \\
\left\|f \underset{T_{1}, T_{2}, T_{1}}{*} g\right\|_{1} & \leq \sqrt{\frac{5}{\pi}}\|f\|_{1}\|g\|_{1} ; \\
\left(T_{1}\left(f \underset{T_{1}, T_{2}, T_{2}}{*} g\right)\right)(n) & =\left(T_{2} f\right)(x)\left(T_{2} g\right)(n) \text { for } n \in \mathbb{Z}, \\
\left\|f \underset{T_{1}, T_{2}, T_{2}}{*} g\right\|_{1} & \leq \sqrt{\frac{5}{\pi}}\|f\|_{1}\|g\|_{1} .
\end{aligned}
$$

Proof. We first prove the theorem for the convolution (3.1). It is easily proven that

$$
\begin{aligned}
& {\left[\frac{1}{2} \cos (n u)-i \sin (n u)\right]\left[\frac{1}{2} \cos (n v)-i \sin (n v)\right]} \\
& =\frac{1}{8}\left\{7\left[\frac{1}{2} \cos (n(u+v))-i \sin (n(u+v))\right]\right. \\
& -3\left[\frac{1}{2} \cos (n(u-v))-i \sin (n(u-v))\right] \\
& -3\left[\frac{1}{2} \cos (n(-u+v))-i \sin (n(-u+v))\right] \\
& \left.+3\left[\frac{1}{2} \cos (n(-u-v))-i \sin (n(-u-v))\right]\right\} .
\end{aligned}
$$

Using this identity, changing the variable and noting the $2 \pi$-periodicity of $f$, we have

$$
\begin{aligned}
&\left(T_{1} f\right)(n)\left(T_{1} g\right)(n)=\frac{5}{4 \pi} \int_{0}^{2 \pi} \int_{0}^{2 \pi}\left[\frac{1}{2} \cos (n u)-i \sin (n u)\right] \times \\
& \quad\left[\frac{1}{2} \cos (n v)-i \sin (n v)\right] f(u) g(v) d u d v \\
&=\frac{5}{8(4 \pi)} \int_{0}^{2 \pi} \int_{0}^{2 \pi}\left\{7\left[\frac{1}{2} \cos (n(u+v))-i \sin (n(u+v))\right]\right. \\
&-3\left[\frac{1}{2} \cos (n(u-v))-i \sin (n(u-v))\right] \\
&-3\left[\frac{1}{2} \cos (n(-u+v))-i \sin (n(-u+v))\right] \\
&\left.+3\left[\frac{1}{2} \cos (n(-u-v))-i \sin (n(-u-v))\right]\right\} f(u) g(v) d u d v \\
&= \frac{5}{8(4 \pi)} \int_{0}^{2 \pi} g(v)\left\{\int_{v}^{2 \pi+v} 7\left[\frac{1}{2} \cos (n x)-i \sin (n x)\right] f(x-v) d x\right. \\
&-\int_{-v}^{2 \pi-v} 3\left[\frac{1}{2} \cos (n x)-i \sin (n x)\right] f(x+v) d x \\
&+\int_{v}^{-2 \pi+v} 3\left[\frac{1}{2} \cos (n x)-i \sin (n x)\right] f(-x+v) d x
\end{aligned}
$$




$$
\begin{aligned}
& \left.-3 \int_{-v}^{-2 \pi-v} 7\left[\frac{1}{2} \cos (n x)-i \sin (n x)\right] f(-x-v) d x\right\} d v \\
= & \frac{5}{8(4 \pi)} \int_{0}^{2 \pi} g(v)\left\{\int_{v}^{2 \pi+v} 7\left[\frac{1}{2} \cos (n x)-i \sin (n x)\right] f(x-v) d x\right. \\
& -\int_{-v}^{2 \pi-v} 3\left[\frac{1}{2} \cos (n x)-i \sin (n x)\right] f(x+v) d x \\
& -\int_{-2 \pi+v}^{v} 3\left[\frac{1}{2} \cos (n x)-i \sin (n x)\right] f(-x+v) d x \\
& \left.+3 \int_{-2 \pi-v}^{-v} 7\left[\frac{1}{2} \cos (n x)-i \sin (n x)\right] f(-x-v) d x\right\} d v . \\
= & \frac{1}{2} \sqrt{\frac{5}{\pi}} \int_{0}^{2 \pi}\left[\frac{1}{2} \cos (n x)-i \sin (n x)\right]\left\{\frac{1}{16} \sqrt{\frac{5}{\pi}} \int_{0}^{2 \pi}[7 f(x-v)\right. \\
& -3 f(x+v)-3 f(-x+v)+3 f(-x-v)] g(v) d v\} d x \\
= & T_{1}(f * * g)(n) .
\end{aligned}
$$

Now, let us prove the norm inequality

$$
\begin{aligned}
\int_{0}^{2 \pi}\left|\left(f \underset{T_{1}}{*} g\right)(x)\right| d x \leq & \frac{1}{16} \sqrt{\frac{5}{\pi}} \int_{0}^{2 \pi} \int_{0}^{2 \pi}[7|f(x-v)|+3|f(x+v)| \\
& +3|f(-x+v)|+3|f(-x-v)|]|g(v)| d v d x \\
= & \frac{16}{16} \sqrt{\frac{5}{\pi}} \int_{0}^{2 \pi} \int_{0}^{2 \pi}\left|f(y)\left\|g(u) \mid d v d y=\sqrt{\frac{5}{\pi}}\right\| f\left\|_{1}\right\| g \|_{1} .\right.
\end{aligned}
$$

We ignore the proofs of the other convolutions as they can be completed in the same way as above, by using corresponding identities. The proof is completed. $T_{2}$.

As a second step in our method, we define four new convolutions associated with the operator

Definition 4. For any two functions $f, g$ that are Lebesgue integrable on $[0,2 \pi]$, being $f$ a function defined on $\mathbb{R}$ and $2 \pi$-periodic, we define four finite Fourier-type convolutions as follows:

$$
\begin{aligned}
& \left(f \underset{T_{2}}{*} g\right)(x):=\frac{1}{16} \sqrt{\frac{5}{\pi}} \int_{0}^{2 \pi}[7 f(x-v)-3 f(x+v)-3 f(-x+v) \\
& +3 f(-x-v)] g(v) d v ; \\
& \left(f_{T_{2}, T_{2}, T_{1}}^{*} g\right)(x):=\frac{1}{16} \sqrt{\frac{5}{\pi}} \int_{0}^{2 \pi}[-3 f(x-v)+7 f(x+v)+3 f(-x+v) \\
& -3 f(-x-v)] g(v) d v ; \\
& \left(f_{T_{2}, T_{1}, T_{2}}^{*} g\right)(x):=\frac{1}{16} \sqrt{\frac{5}{\pi}} \int_{0}^{2 \pi}[-3 f(x-v)+3 f(x+v)+7 f(-x+v) \\
& -3 f(-x-v)] g(v) d v ; \\
& \left(f_{T_{2}, T_{1}, T_{1}}^{*} g\right)(x):=\frac{1}{16} \sqrt{\frac{5}{\pi}} \int_{0}^{2 \pi}[3 f(x-v)-3 f(x+v)-3 f(-x+v) \\
& +7 f(-x-v)] g(v) d v \text {. }
\end{aligned}
$$


The next theorem introduces the relation of those convolutions with the previously considered operators.

Theorem 3.2 (Convolution theorem). If $f, g$ are Lebesgue integrable on $[0,2 \pi]$ and $f$ is a function defined on $\mathbb{R}$ and $2 \pi$-periodic, then each one of the multiplications introduced in Definition 4 has its factorization identity associated with the integral operators $T_{1}, T_{2}$ and the corresponding norm inequality:

$$
\begin{aligned}
\left(T_{2}\left(f \underset{T_{2}}{*} g\right)\right)(n) & =\left(T_{2} f\right)(n)\left(T_{2} g\right)(n) \text { for } n \in \mathbb{Z}, \\
\left\|f \underset{T_{2}}{*} g\right\|_{1} & \leq \sqrt{\frac{5}{\pi}}\|f\|_{1}\|g\|_{1} ; \\
\left(T_{2}\left(f_{T_{2}, T_{1}, T_{1}}^{*} g\right)\right)(n) & =\left(T_{1} f\right)(x)\left(T_{1} g\right)(n) \text { for } n \in \mathbb{Z}, \\
\left\|f_{T_{2}, T_{1}, T_{1}}^{*} g\right\|_{1} & \leq \sqrt{\frac{5}{\pi}}\|f\|_{1}\|g\|_{1} ; \\
\left(T_{2}\left(f_{T_{2}, T_{1}, T_{2}}^{*} g\right)\right)(n) & =\left(T_{1} f\right)(x)\left(T_{2} g\right)(n) \text { for } n \in \mathbb{Z}, \\
\left\|f_{T_{2}, T_{1}, T_{2}}^{*} g\right\|_{1} & \leq \sqrt{\frac{5}{\pi}}\|f\|_{1}\|g\|_{1} ; \\
\left(T_{2}\left(f_{T_{2}, T_{2}, T_{1}}^{*} g\right)\right)(n) & =\left(T_{2} f\right)(x)\left(T_{1} g\right)(n) \text { for } n \in \mathbb{Z}, \\
\left\|f \underset{T_{2}, T_{2}, T_{1}}{*} g\right\|_{1} & \leq \sqrt{\frac{5}{\pi}}\|f\|_{1}\|g\|_{1} .
\end{aligned}
$$

We will omit the proof of Theorem 3.2 because it is very similar to the proof of Theorem 3.1 . Due to the fact of $L^{2}([0,2 \pi]) \subset L^{1}([0,2 \pi])$, we can state the following theorem.

Theorem 3.3. Suppose that $f, g$ are Lebesgue integrable on $[0,2 \pi]$ and $f$ is a function defined on $\mathbb{R}$ and $2 \pi$-periodic. If $f, g \in L^{2}([0,2 \pi])$, then the following norm inequality holds:

$$
\|f \underset{T}{*} g\|_{2} \leq \frac{\sqrt{190}}{4}\|f\|_{2}\|g\|_{2},
$$

where ${ }_{T}^{*}$ represents anyone of the convolutions 3.1 -3.2 and 3.10-3.11.

Proof. We will just prove the theorem for the convolution (3.1). For the other ones, we proceed in a similar way.

By the Cauchy-Schwarz inequality, we have $(a+b+c+d)^{2} \leq 4\left(a^{2}+b^{2}+c^{2}+d^{2}\right)$ and so,

$$
\begin{aligned}
\left|\left(f \underset{T_{1}}{*} g\right)(x)\right|^{2}= & \left(\frac{1}{16} \sqrt{\frac{5}{\pi}}\right)^{2}\left|\int_{0}^{2 \pi}[7 f(x-v)-3 f(x+v)-3 f(-x+v)+3 f(-x-v)] g(v) d v\right|^{2} \\
\leq & \frac{5}{256 \pi} \int_{0}^{2 \pi}|g(v)|^{2} d v \int_{0}^{2 \pi}|7 f(x-v)-3 f(x+v)-3 f(-x+v)+3 f(-x-v)|^{2} d v \\
\leq & \frac{5}{256 \pi} \int_{0}^{2 \pi}|g(v)|^{2} d v \int_{0}^{2 \pi} 4\left[49|f(x-v)|^{2}+9|f(x+v)|^{2}\right. \\
& \left.+9|f(-x+v)|^{2}+9|f(-x-v)|^{2}\right] d v=\frac{95}{16 \pi}\|f\|_{2}^{2}\|g\|_{2}^{2} .
\end{aligned}
$$

This implies that

$$
\left\|f \underset{T_{1}}{*} g\right\|_{2}=\left(\int_{0}^{2 \pi}\left|\left(f \underset{T_{1}}{*} g\right)(x)\right|^{2} d x\right)^{\frac{1}{2}} \leq \frac{\sqrt{190}}{4}\|f\|_{2}\|g\|_{2},
$$


which proves the proposition.

\section{ON THE SOLUTiON OF THE INTEGRAL EQUATION}

This section is devoted to the solvability of equation (1.1), where $p$ and $q$ are given $2 \pi$-periodic one-variable functions. In particular, the kernel $K(x, u)$ contains two terms: a convolution $p(x-u)$ and a cross-correlation $q(x+u)$, which has many applications on pattern recognition, single particle analysis, electron tomography, averaging, etc..

The key tool for investigating (1.1) are the convolutions constructed in Section 3 . We will see the fact that such set of eight convolutions is very useful for our purposes. From our point of view, some subgroup of such convolution set may be used for other particular purposes.

Let us write:

$$
\begin{aligned}
A(n) & :=\lambda+\frac{1}{4} \sqrt{\frac{\pi}{5}}\left[13\left(T_{1} p\right)(n)+3\left(T_{2} p\right)(n)+3\left(T_{1} q\right)(n)-3\left(T_{2} q\right)(n)\right] \\
B(n) & :=\frac{1}{4} \sqrt{\frac{\pi}{5}}\left[3\left(T_{1} p\right)(n)-3\left(T_{2} p\right)(n)+13\left(T_{1} q\right)(n)+3\left(T_{2} q\right)(n)\right] \\
D(n) & :=A(n) A(-n)-B(n) B(-n) \\
D_{1}(n) & :=A(-n)\left(T_{1} f\right)(n)-B(n)\left(T_{2} f\right)(n) \\
D_{2}(n) & :=A(n)\left(T_{2} f\right)(n)-B(-n)\left(T_{1} f\right)(n) .
\end{aligned}
$$

Theorem 4.1. Assume that the functions $p, q$ are $2 \pi$-periodic and piecewise continuous on the interval $[0,2 \pi]$, and $f \in L^{2}([0,2 \pi])$.

(i) If $\lambda \neq 0$, then there exists an integer $K^{*}$ such that $D(n) \neq 0$, for every $n \geq K^{*}$.

(ii) If $D(n) \neq 0$, for every $n \in \mathbb{N}_{0}$, then equation (1.1) has a unique solution for every $f \in$ $L^{2}([0,2 \pi])$, which is given by

$$
\varphi(x)=\sum_{n \in \mathbb{Z}} \frac{D_{1}(n)}{D(n)} \mathscr{E}(n x) .
$$

Proof. (i) By the Riemann-Lebesgue lemma for $T_{1}$ and $T_{2}$, we deduce that

$$
\lim _{n \rightarrow \infty} D(n)=\lambda^{2} \neq 0 \text {. }
$$

Hence, there exists an integer $K^{*} \in \mathbb{N}$ such that $D(n) \neq 0$, for all $n \geq K^{*}$. Item (i) is proved.

(ii) The four numbers $7,-3,-3,3$ appearing in the right-hand sides of the convolution identities (3.1)-(3.4) constitute a symmetric matrix in the form

$$
M:=\left(\begin{array}{cccc}
7 & -3 & -3 & 3 \\
-3 & 7 & 3 & -3 \\
-3 & 3 & 7 & -3 \\
3 & -3 & -3 & 7
\end{array}\right),
$$

from which it follows $\operatorname{det}(M)=1024 \neq 0$. The matrix $M$ is defined as follows: each line of the matrix is built by using the coefficients of the kernel of each convolution (accordingly with the exhibited order), this is, the first line consists of the kernel coefficients of the first convolution in the order that appears in its definition, the second line consists of the kernel coefficients of the second convolution in the same order, and so on. 
This method can be generalized for all the coefficients of the operator such that $\operatorname{det}(M) \neq 0$. Therefore, replacing $g$ with $\varphi$ and $f$ with $p$ in $3.1-3.4$ and converting those expressions, we obtain

$$
\begin{aligned}
\int_{0}^{2 \pi} p(x-u) \varphi(u) d u=\frac{1}{4} \sqrt{\frac{\pi}{5}}\left[13\left(p_{T_{1}}^{*} \varphi\right)(x)+3\left(p_{T_{1}, T_{1}, T_{2}}^{*} \varphi\right)(x)\right. \\
\left.+3\left(p_{T_{1}, T_{2}, T_{1}}^{*} \varphi\right)(x)-3\left(p_{T_{1}, T_{2}, T_{2}}^{*} \varphi\right)(x)\right] .
\end{aligned}
$$

Again, replacing $g$ with $\varphi$ and $f$ with $q$ and converting those expressions as above, we get

$$
\begin{aligned}
\int_{0}^{2 \pi} q(x+u) \varphi(u) d u=\frac{1}{4} \sqrt{\frac{\pi}{5}}\left[3\left(q_{T_{1}}^{*} \varphi\right)(x)+13\left(q_{T_{1}, T_{1}, T_{2}}^{*} \varphi\right)(x)\right. \\
\left.-3\left(q_{T_{1}, T_{2}, T_{1}}^{*} \varphi\right)(x)+3\left(q_{T_{1}, T_{2}, T_{2}}^{*} \varphi\right)(x)\right] .
\end{aligned}
$$

Applying $T_{1}$ to both sides of these identities and using the factorization identities of the convolutions appeared on the right-hand side, we obtain

$$
\begin{aligned}
& T_{1}\left(\int_{0}^{2 \pi} p(x-u) \varphi(u) d u\right)(n)=\frac{1}{4} \sqrt{\frac{\pi}{5}}\left[13\left(T_{1} p\right)(n)\left(T_{1} \varphi\right)(n)\right. \\
& \left.\quad+3\left(T_{1} p\right)(n)\left(T_{2} \varphi\right)(n)+3\left(T_{2} p\right)(n)\left(T_{1} \varphi\right)(n)-3\left(T_{2} p\right)(n)\left(T_{2} \varphi\right)(n)\right] \\
& T_{1}\left(\int_{0}^{2 \pi} q(x+u) \varphi(u) d u\right)(n)=\frac{1}{4} \sqrt{\frac{\pi}{5}}\left[3\left(T_{1} q\right)(n)\left(T_{1} \varphi\right)(n)\right. \\
& \left.\quad+13\left(T_{1} q\right)(n)\left(T_{2} \varphi\right)(n)-3\left(T_{2} q\right)(n)\left(T_{1} \varphi\right)(n)+3\left(T_{2} q\right)(n)\left(T_{2} \varphi\right)(n)\right] .
\end{aligned}
$$

Analogously, we convert the convolution 3.10 -3.13 to have

$$
\begin{aligned}
& T_{2}\left(\int_{0}^{2 \pi} p(x-u) \varphi(u) d u\right)(n)=\frac{1}{4} \sqrt{\frac{\pi}{5}}\left[13\left(T_{2} p\right)(n)\left(T_{2} \varphi\right)(n)\right. \\
& \left.\quad+3\left(T_{2} p\right)(n)\left(T_{1} \varphi\right)(n)+3\left(T_{1} p\right)(n)\left(T_{2} \varphi\right)(n)-3\left(T_{1} p\right)(n)\left(T_{1} \varphi\right)(n)\right] ; \\
& T_{2}\left(\int_{0}^{2 \pi} q(x+u) \varphi(u) d u\right)(n)=\frac{1}{4} \sqrt{\frac{\pi}{5}}\left[3\left(T_{2} q\right)(n)\left(T_{2} \varphi\right)(n)\right. \\
& \left.\quad+13\left(T_{2} q\right)(n)\left(T_{1} \varphi\right)(n)-3\left(T_{1} q\right)(n)\left(T_{2} \varphi\right)(n)+3\left(T_{1} q\right)(n)\left(T_{1} \varphi\right)(n)\right] .
\end{aligned}
$$

To prove the uniqueness of the solution of equation 1.1 , we invoke the Fredholm alternative theorem. Suppose that the homogeneous equation corresponding to equation (1.1) has a solution $\varphi_{*} \in L^{2}([0,2 \pi])$, this is,

$$
\lambda \varphi_{*}(x)+\int_{0}^{2 \pi}[p(x-u)+q(x+u)] \varphi_{*}(u) d u=0 .
$$

Applying $T_{1}$ and $T_{2}$ to both sides of this equation and using identities (4.7)-4.10), we obtain the following system of two linear equations

$$
\left\{\begin{array}{l}
A(n)\left(T_{1} \varphi_{*}\right)(n)+B(n)\left(T_{2} \varphi_{*}\right)(n)=0 \\
B(-n)\left(T_{1} \varphi_{*}\right)(n)+A(-n)\left(T_{2} \varphi_{*}\right)(n)=0 .
\end{array}\right.
$$

Since $D(n) \neq 0$, for every $n \in \mathbb{N}_{0}$, we obtain $\left(T_{1} \varphi_{*}\right)(n)=\left(T_{2} \varphi_{*}\right)(n)=0$, for $n \geq 0$. Due to the uniqueness theorem of the operators $T_{1}$ and $T_{2}$, we obtain that $\varphi_{*}=0$. Thus, the homogeneous 
equation associated with the equation (1.1) has only the trivial solution and so, by the Fredholm alternative theorem, equation $(1.1)$ has a unique non-trivial solution, provided $0 \neq f \in L^{2}([0,2 \pi])$.

Now, we will perform the solution formula (4.6). Suppose that $\varphi \in L^{2}([0,2 \pi])$ is fulfilling [1.1]. Proceeding in a similar way as used for the homogeneous equation, we obtain the following system

$$
\left\{\begin{array}{l}
A(n)\left(T_{1} \varphi\right)(n)+B(n)\left(T_{2} \varphi\right)(n)=\left(T_{1} f\right)(n) \\
B(-n)\left(T_{1} \varphi\right)(n)+A(-n)\left(T_{2} \varphi\right)(n)=\left(T_{2} f\right)(n) .
\end{array}\right.
$$

Since $D(n) \neq 0$ for every $n \geq 0$, this system has a unique solution given by

$$
\left(T_{1} \varphi\right)(n)=\frac{D_{1}(n)}{D(n)}, \quad\left(T_{2} \varphi\right)(n)=\frac{D_{2}(n)}{D(n)}=\frac{D_{1}(-n)}{D(n)}=\left(T_{1} \varphi\right)(-n)
$$

for $n=0,1, \ldots$. Taking the inverse transform of $T_{1}$, we obtain

$$
\varphi(x)=\sum_{n \in \mathbb{Z}} \frac{D_{1}(n)}{D(n)} \mathscr{E}(n x)
$$

which belongs to $L^{2}([0,2 \pi])$ and fulfills $(1.1)$ for almost every $x \in[0,2 \pi]$, where

$$
\|\varphi\|_{2}^{2}=\frac{\pi}{2}\left|\hat{f}_{c}(0)\right|^{2}+\sum_{0 \neq n \in \mathbb{Z}}\left|\frac{D_{1}(n)}{D(n)}\right|^{2}<\infty .
$$

The theorem is proved.

The solution 4.6 can be called a Shannon-type sampling formula concerning the function $\mathcal{E}$. In other words, we can cover all signals from the knowledge of the information at the samples $\frac{D_{1}(n)}{D(n)}$.

\section{EXAMPLES AND COMPARISON}

In this section, we present some examples of integral equations that we can solve by using the above integral transforms and convolutions and that are not possible to solve by using previously published work. From our point of view, the non symmetry of the coefficients in the transforms $T_{1}$ and $T_{2}$ have a significant effect in this situation.

Example 1. Let us consider the equation

$$
2 \varphi(x)+\int_{0}^{2 \pi}[\operatorname{cas}(x-u)+H(x+u)] \varphi(u) d u=2,
$$

that is under the conditions of Theorem 4.1, where $p(x)=\operatorname{cas}(x):=\cos (x)+\sin (x)$ and $q(x)=$ $H(x)$ stands for the Heaviside function. If we use the Hartley transform as in the paper [2], then $D(n)=0$ for $n=0$ (for the corresponding $D$ defined in the sense of the present work). In view of this, it follows that the reasoning in the just mentioned paper does not work for this equation. However, using the operators $T_{1}$ and $T_{2}$, we obtain by our method

$$
D(n)= \begin{cases}4+2 \pi+2 \pi^{2}, & n=1 \\ 4\left(1-\pi^{2}\right), & n=0 \\ 4, & n \neq 0 \wedge n \neq 1 .\end{cases}
$$

This implies $D(n) \neq 0$ for all $n \in \mathbb{Z}$. As a result, we can apply Theorem 4.1 for obtaining the unique solution given by 4.6 .

Example 2. Let us consider the following equation

$$
\varphi(x)+\int_{0}^{2 \pi}\left[\sin \left(m_{1}(x-u)\right)+\cos \left(m_{2}(x+u)\right)\right] \varphi(u) d u=\cos (x),
$$


with $m_{1} \neq m_{2}$, that is under the conditions of Theorem 4.1. where $p(x)=\sin \left(m_{1} x\right)$ and $q(x)=$ $\cos \left(m_{2} x\right)$. If we use the Hartley transform as applied in [2], then $D(n)=0$ for $n=m_{2}$ (with the $D(n)$ of that paper). So, the approach to this equation via the Hartley transform, as exposed in [2], does not work for analyzing the solvability and obtaining the eventual unique solution of the present equation.

Nevertheless, using the operators $T_{1}$ and $T_{2}$, our method works in this case since

$$
D(n)= \begin{cases}1+\frac{25}{16} \pi^{2}, & n=m_{1} \\ 1-\pi^{2}, & n=m_{2} \\ 1, & n \neq m_{1} \wedge n \neq m_{2} .\end{cases}
$$

In particular, some simple computations give

$$
D_{1}(n)=D_{2}(n)= \begin{cases}(1-\pi) \frac{\sqrt{5 \pi}}{4}, & n=m_{2} \\ 0, & n \neq m_{2} .\end{cases}
$$

and the solution $\varphi(x)=\frac{\cos \left(m_{2} x\right)}{1+\pi}$.

Example 3. Consider the equation

$$
\frac{3}{4} \varphi(x)+\int_{0}^{2 \pi}[\cos (x-u)+(x+u) \sin (x+u)] \varphi(u) d u=1,
$$

that is under the conditions of Theorem 4.1. where $p(x)=\cos (x)$ and $q(x)=x \sin (x)$. The use of the Hartley transform in [2] also does not work in this case since their $D(n)=0$ for $n=3$; i.e., with the method of that paper we cannot analyze the solvability of this equation and also cannot obtain its unique solution.

However, by using the operators $T_{1}$ and $T_{2}$, we obtain

$$
D(n)= \begin{cases}\frac{9}{16}+3 \pi-\pi^{4}, & n=1 \\ \frac{9}{16}-\frac{4 \pi^{2} n^{2}}{\left(n^{2}-1\right)^{2}}, & n \neq 1 .\end{cases}
$$

Therefore, we can apply Theorem 4.1 for obtaining the unique solution of the equation.

Example 4. Consider the following equation

$$
\varphi(x)+\int_{0}^{2 \pi}[\sin (3(x-u))+\cos (x+u)] \varphi(u) d u=\cos (x),
$$

that is under the conditions of Theorem 4.1. Using the operators $T_{1}$ and $T_{2}$, we obtain

$$
\begin{aligned}
& \left(T_{1} q\right)(n)=\left(T_{2} q\right)(n)= \begin{cases}0, & n \neq 1 \\
\frac{\sqrt{5 \pi}}{4}, & n=1 ;\end{cases} \\
& \left(T_{1} p\right)(n)=\left\{\begin{array}{ll}
0, & n \neq 3 \\
-\frac{i \sqrt{5 \pi}}{2}, & n=3 ;
\end{array} \quad\left(T_{2} p\right)(n)= \begin{cases}0, & n \neq 3 \\
\frac{i \sqrt{5 \pi}}{2}, & n=3 .\end{cases} \right.
\end{aligned}
$$

Performing some straightforward computations, we derive:

$$
\begin{gathered}
A(n)= \begin{cases}1-\frac{5 \pi}{4} i, & n=3 \\
1, & n \neq 3 ;\end{cases} \\
A(-n)= \begin{cases}1+\frac{5 \pi}{4} i, & n=3 \\
1, & n \neq 3\end{cases}
\end{gathered}
$$


and

$$
\begin{gathered}
B(n)= \begin{cases}\pi, & n=1 \\
-\frac{3 \pi i}{4}, & n=3 \\
0, & n \neq 1 \wedge n \neq 3 ;\end{cases} \\
B(-n)= \begin{cases}\pi, & n=1 \\
\frac{3 \pi i}{4}, & n=3 \\
0, & n \neq 1 \wedge n \neq 3 .\end{cases}
\end{gathered}
$$

The above identities provide

$$
D(n)= \begin{cases}1-\pi^{2}, & n=1 \\ 1+\pi^{2}, & n=3 \\ 1, & n \neq 1 \wedge n \neq 3\end{cases}
$$

which implies $D(n) \neq 0$ for all $n \in \mathbb{N}_{0}$. We deduce that Theorem 4.1 allow us to characterize the solvability and obtain the unique solution of this integral equation. Namely, we have

$$
\begin{aligned}
& \left(T_{1} f\right)(n)=\left(T_{2} f\right)(n)= \begin{cases}\frac{\sqrt{5 \pi}}{4}, & n=1 \\
0, & n \neq 1,\end{cases} \\
& D_{1}(n)=D_{2}(n)= \begin{cases}(1-\pi) \frac{\sqrt{5 \pi}}{4}, & n=1 \\
0, & n \neq 1 .\end{cases}
\end{aligned}
$$

which provides the solution $\varphi(x)=\frac{\cos (x)}{1+\pi}$.

However, the paper [2] also does not work for this equation. Indeed, obeying the procedures in that paper, we have

$$
\begin{aligned}
& \left(H_{1} p\right)(n)=\left\{\begin{array}{ll}
0, & n \neq 3 \\
\frac{1}{2}, & n=3 ;
\end{array} \quad\left(H_{2} p\right)(n)= \begin{cases}0, & n \neq 3 \\
-\frac{1}{2}, & n=3 .\end{cases} \right. \\
& \left(H_{1} q\right)(n)=\left(H_{2} q\right)(n)= \begin{cases}0, & n \neq 1 \\
\frac{1}{2}, & n=1 .\end{cases}
\end{aligned}
$$

Using some additional computations, we have $A(n)=A(-n)=1$, and

$$
B(n)=\left\{\begin{array}{ll}
1, & n=1,3 \\
0, & n \neq 1 \wedge n \neq 3
\end{array} \quad B(-n)= \begin{cases}1, & n=1 \\
-1, & n=3 \\
0, & n \neq 1 \wedge n \neq 3\end{cases}\right.
$$

We find

$$
D(n)= \begin{cases}0, & n=1 \\ 2, & n=3 \\ 1, & n \neq 1 \wedge n \neq 3\end{cases}
$$

From this it follows $D(1)=0$, which confirms the above conclusion. 


\section{Concluding Remarks}

For convenience of formulation, we write

$$
\begin{aligned}
& \mathbb{S}_{1}:=\left\{-\pi\left[\hat{p}_{c}(n) \mp \hat{q}_{c}(n)\right]: n \in \mathbb{Z}\right\} \\
& \mathbb{S}_{2}:=\left\{-\pi\left[\hat{p}_{c}(n) \mp \hat{q}_{s}(n)\right]: n \in \mathbb{Z}\right\} ; \\
& \mathbb{S}_{3}:=\left\{ \pm \pi \sqrt{\hat{q}_{c}^{2}(n)-\hat{p}_{s}^{2}(n)}: n \in \mathbb{Z}\right\} ; \\
& \mathbb{S}_{4}:=\left\{ \pm \pi \sqrt{\hat{q}_{s}^{2}(n)-\hat{p}_{s}^{2}(n)}: n \in \mathbb{Z}\right\} .
\end{aligned}
$$

We note that the square roots in $\mathbb{S}_{3}$ as well as in $\mathbb{S}_{4}$ are understood in complex sense, i.e. each one of $\mathbb{S}_{3}$ and $\mathbb{S}_{4}$ is just a single-valued branch of the root. In a more general perspective, we are able to state the following proposition.

Proposition 6.1. Suppose that the functions $p$ and $q$ in the equation (1.1) are Lebesgue integrable.

(i) If $p, q$ are even functions, then $D(n) \neq 0$ for all $n \in \mathbb{N}_{0}$ if and only if $\lambda \notin \mathbb{S}_{1}$.

(ii) If $p$ is an even function and $q$ is an odd function, then $D(n) \neq 0$ for all $n \in \mathbb{N}_{0}$ if and only if $\lambda \notin \mathbb{S}_{2}$.

(iii) If $p$ is an odd function and $q$ is an even function, then $D(n) \neq 0$ for all $n \in \mathbb{N}_{0}$ if and only if $\lambda \notin \mathbb{S}_{3}$.

(iv) If $p, q$ are odd functions, then $D(n) \neq 0$ for all $n \in \mathbb{N}_{0}$ if and only if $\lambda \notin \mathbb{S}_{4}$.

Proof. We shall present the proof for Item (i), and leave the proofs for the other cases, since they can be completed in a similar way. For Item (i), we have:

$$
\begin{aligned}
\left(T_{1} p\right)(n) & =\left(T_{2} p\right)(n)=\frac{1}{4} \sqrt{\frac{5}{\pi}} \pi \hat{p}_{c}(n) \\
\left(T_{1} q\right)(n) & =\left(T_{2} q\right)(n)=\frac{1}{4} \sqrt{\frac{5}{\pi}} \pi \hat{p}_{c}(n) \\
A(n) & =A(-n)=\lambda+\pi \hat{p}_{c}(n) \\
B(n) & =B(-n)=\pi \hat{q}_{c}(n)
\end{aligned}
$$

Thus, $D(n)=\left(\lambda+\pi \hat{p}_{c}(n)\right)^{2}-\left(\pi \hat{q}_{c}(n)\right)^{2}$. We derive that $D(n) \neq 0$ if and only if $\lambda \neq \pi\left(-\hat{p}_{c}(n) \pm \hat{q}_{c}(n)\right)$, which proves Item (i). The proposition is proved.

Corresponding to each one of cases of the given kernel functions $p, q$ in Items (i), (ii), (iii) and (iv), the left-hand side of the integral equation (1.1) defines a Fredholm integral operator acting in the Hilbert space $L^{2}([0,2 \pi])$; say $T_{p q}:=I+K_{p q}$.

We state the following theorem which is in some sense a reformulation of Proposition 6.1 .

Theorem 6.2. The point spectrum of the Fredholm integral operator $T_{p q}$ is the set $\mathbb{S}_{1}, \mathbb{S}_{2}, \mathbb{S}_{3}$ and $\mathbb{S}_{4}$ where the kernel functions $p, q$ are given by that in Item (i), (ii), (iii), and (iv) of Proposition 6.1. respectively.

So, there exists an enough large class of Lebesgue integrable $2 \pi$-periodic functions for the Wiener-Hopf and Hankel kernels $p, q$ to which the finite integral transforms and series of Fouriertype provide an effective tool for investigating the solvability and explicit solution of the equation. 
The $L^{2}$-series solution (4.6), in one side, provides helpful procedures in approximation and numerical computations, in another side, that can be viewed as a Shannon's sampling formula with the given data being of the Wiener-Hopf and Hankel form. This may be of interest in digital processing by admitting the linear time-invariant system in sense of those kernels. Theoretically, Theorem 6.2 shows a particular class of Fredholm integral operators defined on the Hilbert space $L^{2}([0,2 \pi])$ together with their explicit point spectrums.

\section{REFERENCES}

[1] Anderson, B. D. O.-Kailath, T.: Fast Algorithms for the Integral Equations of the Inverse Scatting Problem, Integral Equations Operator Theory 1 (1978), 132-136.

[2] Anh, P. K.-Tuan, N. M.-Tuan, P. D.: The finite Hartley new convolutions and solvability of the integral equations with Toeplitz plus Hankel kernels, J. Math. Anal. Appl. 397 (2013), 537-549.

[3] Anh, P. K.-Castro, L. P.-Thao, P. T.-Tuan, N. M.: Two New Convolutions for the Fractional Fourier Transform, Wireless Pers. Commun. 92 (2017), 623-637.

[4] Bogveradze, G.-Castro, L. P.: Toeplitz plus Hankel operators with infinite index, Integral Equations Operator Theory 62(1) (2008), 43-63.

[5] Chanda, K.-Sabatier, P. C.: Inverse Problems in Quantum Scattering Theory, Springer-Verlag, New-York, 1977.

[6] Castro, L. P.-Guerra, R. C.-Tuan, N. M.: Heisenberg uncertainty principles for an oscillatory integral operator, American Institute of Physics, AIP Proceedings 1798(1) (2017), 020037, 10pp., http://dx.doi.org/10.1063/1.4972629

[7] Castro, L. P.-Guerra, R. C.-Tuan, N. M.: On Wieners Tauberian theorems and convolution for oscillatory integral operators, Turkish J. Math. 43(3) (2019), 11241147.

[8] Castro, L. P.-Itou, H.-Saitoh, S.: Numerical solutions of linear singular integral equations by means of Tikhonov regularization and reproducing kernels, Houston J. Math., 38(4) (2012), 1261-1276.

[9] Castro, L. P.-Minh, L. T.-Tuan, N. M.: New convolutions for quadratic-phase Fourier integral operators and their applications, Mediterr. J. Math. 15(13) (2018), 1-17.

[10] Castro, L. P.-Rojas, E. M.: Explicit solutions of Cauchy singular integral equations with weighted Carleman shift, J. Math. Anal. Appl. 371 (2010), 128-133.

[11] Castro, L. P.-Rojas, E. M.-Saitoh, S.-Tuan, N. M.-Tuan, P. D.: Solvability of singular integral equations with rotations and degenerate kernels in the vanishing coefficient case, Anal. Appl. (Singap.) 13(1) (2015), $1-21$.

[12] Castro, L. P.-Saitoh, S.: New convolutions and norm inequalities, Math. Inequal. Appl. 12(3) (2012), 707716.

[13] Debnath, L.-Bhatta, D.: Integral transforms and their applications, Second Edition, Chapman \& Hall/CRC, Boca Raton, 2007.

[14] Duc, D. T.-Nha, N. D. V.: Norm inequalities for new convolutions and their applications, Appl. Anal. Discrete Math. 9 (2015), 168-179.

[15] Kailath, T.-Levy, B.-Ljung, L.-Morf, M.: Fast time-invariant implementations of Gaussian signal detectors, IEEE Trans. Inform. Theory 24(4) (1978), 469-477.

[16] Katznelson, Y.: An Introduction to Harmonic Analysis, Cambridge University Press, Cambridge, 2004.

[17] Nha, N. D. V.-Duc, D. T.-Tuan, V. K.: Weighted $L_{p}$-norm inequalities for various convolution type transformations and their applications, Armen. J. Math. 1(4) (2008), 1-18.

[18] Stein, E. M.-Shakarchi, R.: Fourier analysis. An introduction. Princeton Lectures in Analysis, I, Princeton University Press, Princeton and Oxford, 2007.

[19] Tsitsiklis, J. N.-Levy, B. C.: Integral Equations and Resolvents of Toeplitz plus Hankel Kernels. In: Technical Report LIDS-P-1170, Laboratory for Information and Decision Systems, M.I.T., 1981.

[20] Zygmund, A.: Trigonometric series, Third edition: Volumes I \& II combined, Cambridge University Press, Cambridge, 2002.

* Cidma - Center for Research and Development in Mathematics and Applications, Department of Mathematics, University of Aveiro, 3810-193 Aveiro, Portugal.

E-mail address: castro@ua.pt

** Department of Mathematics, College of Education, Viet Nam National University, G7 Build., 144 Xuan Thuy Rd., Cau Giay Dist., Hanoi, Vietnam.

E-mail address: tuannm@hus.edu.vn 